\title{
Infrared thermography as applied to the studies of cardiovascular system in rats
}

\author{
by B. G. Vainer ${ }^{\star}$, V. I. Baranov** and E. G. Vergunov***
}

* Rzhanov Institute of Semiconductor Physics SB RAS, Novosibirsk State University; 13 Lavrentyev av., Novosibirsk, 630090, Russia, BGV@isp.nsc.ru

** State Research Institute of Physiology and Fundamental Medicine SB RAMS, Novosibirsk, Russia, v.i.baranov@physiol.ru

*** Novosibirsk State Pedagogical University, Novosibirsk, Russia, vergounov@gmail.com

Interventional FPA-based infrared thermography (IRT) [1] is applied to the investigation of transient cardiovascular reactions and blood flow change emerging in response to external mechanical and/or thermal impacts in laboratory albino rats. Synchronously with the infrared camera TKVr-IFP/SVIT, the technique enabling measurements of cardiac R-R intervals and heart rate variability [2] was applied as an alternative control method accompanying thermal imaging. Electrocardiogram (ECG) recording was realized using hardware and software system "Veda Puls" manufactured by LLC Biokvant (Novosibirsk, Russia). Sampling frequency of the ECG was $500 \mathrm{~Hz}$.

Narcotized laboratory rats were placed into the special box (figure 1) designed for IRT-based investigation of small animals and described in [1]. The use of this box helped us to avoid many bothering artefacts typical of highprecision thermal studies. Photograph (figure 2) and thermograms (figure 3) provide an insight into the typical setting accompanying the study. They demonstrate a rat undergoing the interventional IRT-based examination. We proposed and successfully tested the use of thermoelectric (Peltier) cooler as a convenient tool appropriate for a lot of physiological applications where a local heating or cooling of outer parts of an animal or human body is necessary.

Local and systemic cardiovascular manifestations in rats were investigated in details. Vascular reactions were monitored during and after local and/or whole body heating, local cooling, crossclamping of the main vessels of a paw and creating artificial ischemia in the rat tail.

Owing to quantitative FPA-based IRT, we were fortunate to disclose several intriguing physiological phenomena in rats. First of all, it was revealed that, even in the rat's calm sleeping condition, its blood supply exhibits activity which is not always definitely explainable. Figure 4 demonstrates temporal alterations in the temperature of different parts of the

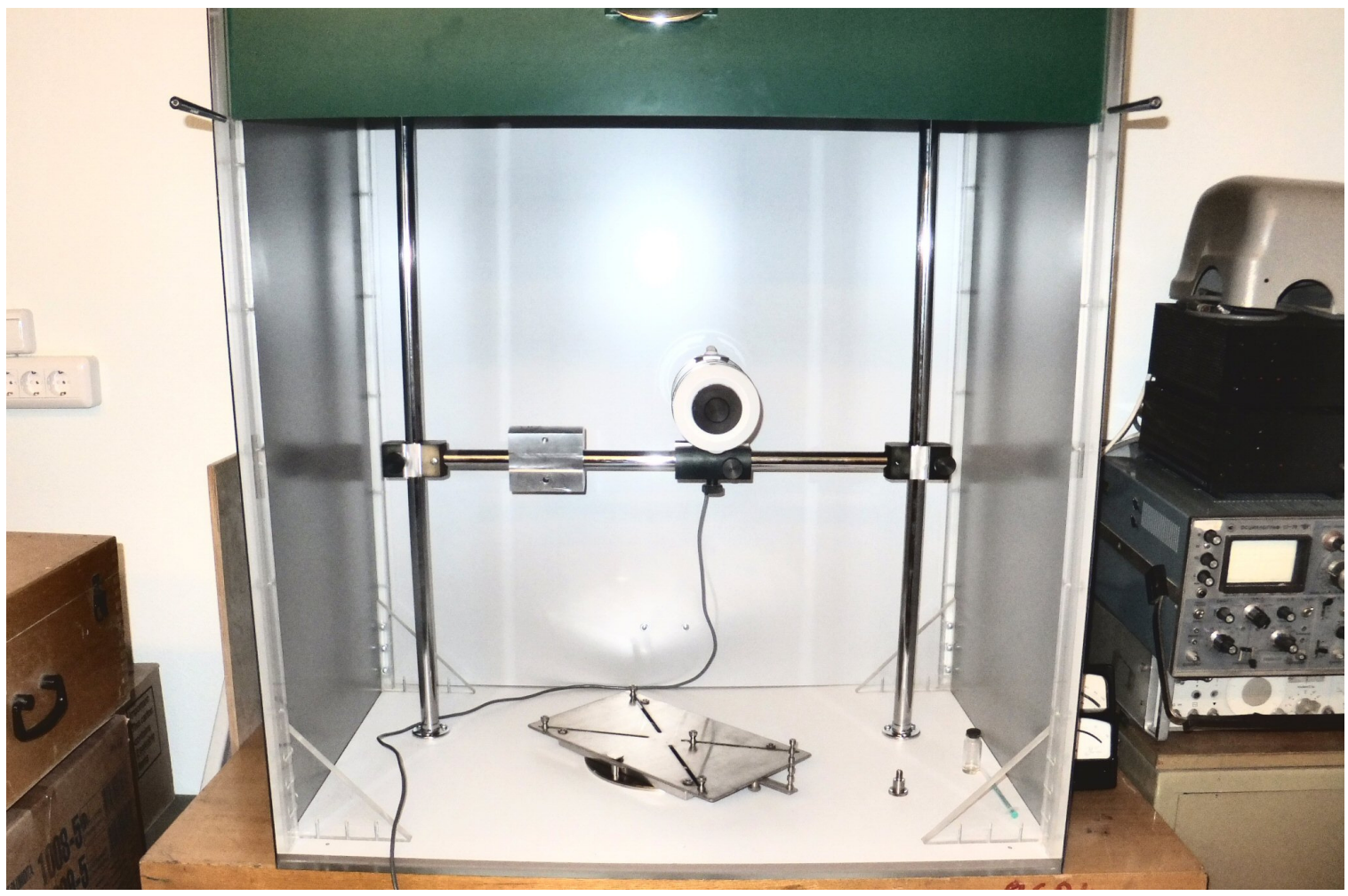

Fig. 1. A special shielding box equipped with mechanical support for infrared camera and universal rotating table for small laboratory animals. The infrared camera (TKVr-IFP/SVIT) is shown in the off (horizontal) position. 
rat's body while the investigated animal simply sleeps and is not disturbed. It can be seen that the temperature of the front left limb remains without remarkable changes while other parts of body subjected to control demonstrate a dramatic $\left(5-10^{\circ} \mathrm{C}\right)$ change in temperature. This temperature change may be rather gradual (hind right limb) or abrupt of the threshold type (front right and hind left limbs; base, middle and tip of tail). The step-like stage of temperature change may occur in the different parts of body rather spontaneously and quite surprisingly. In figure 4, one can find the emergence of these steps in the front right limb at the time $t \approx 700 \mathrm{~s}$, in hind left limb, base and middle of the tail - at $t \approx 2200 \mathrm{~s}$, in the tip of tail - at $\mathrm{t} \approx 4800 \mathrm{~s}$.

In figure 4, the correlation between the temperature change and the trend of heart rate is clearly seen just at the time $t \approx 600-700 \mathrm{~s}$ when the front right limb steeply increases in temperature and the heart rate sharply decreases. One more observation can be made from figure 4 . It is seen that the undulating shape of the heart-rate curve after its sharp decrease is opposite in phase of the front-right-limb temperature undulation: heart rate is decreased - temperature is increased.

The latter phenomenon was also observed in different subjects and different situations. For example, this can be clearly seen in figure 5 which demonstrates that the heating of a hind limb results in quite a remarkable increase of the rat's heart rate with the corresponding synchronous decrease disinterested limbs in temperature. At the same time, it should be noted that the temperature decrease in these experiments took place just when the temperature of heated panel of the Peltier cooler exceeded 42 degrees centigrade. At lower temperatures of the panel, there were no material change in temperature of the limbs other than the heated one. This fact gives ground to two hypotheses. First, $42{ }^{\circ} \mathrm{C}$ is a thermal threshold of pain sensitivity in rats. Second, despite narcotism, a living organism persists to feel pain.

Figure 6 represents a period of relaxation of the rat after double heating of the hind right limb exhibited in figure 5. Here we can see again that the heart rate and limb temperature are in the antiphase. An interesting but not yet statistically valid fact is that all limbs demonstrate dramatic short-term increase in temperature lasting about 3 minutes and having a time interval of about $1000 \mathrm{~s}$. In other words, we observe a sui generis deferred systemic reaction of the organism to the applied external two impacts. It should be noted that heating of the hind right paw above $42{ }^{\circ} \mathrm{C}$ gave birth to local edema and to the lasting elevated temperature (about $35^{\circ} \mathrm{C}$ ) in this limb. In spite of this side effect, the time dependence of temperature in this paw, nevertheless, exhibits modulation in the most pronounced time zones of the organism alterations (at $\mathrm{t}=800 \mathrm{~s}, 1850 \mathrm{~s}, 2400 \mathrm{~s}$ ) that can be seen in figure 6 .

Peltier cooler allowed the paw to cool down rapidly. At the same time, the limb fast cooling to the temperature of about $14{ }^{\circ} \mathrm{C}$ did not demonstrate as significant systemic alterations in the rat's organism as those we observed in the heating regime. It is illustrated in figure 7 . We were fortunate in registering a specific regulative reaction all the same. Immediately after cooling, the wavy variation of temperature with the period of about $33 \mathrm{~s}$ was detected in all limbs that is clearly represented in figure 7 . As this took place, the tail remained indifferent to this kind of impact. The latter fact proves that the observed phenomenon cannot be explained as a hardware-generated effect.

The crossclamping of the rat's limb or tail vessels served as another kind of external impact to the organism. Similar intervention was previously put to the test in humans using the crossclamping of their shoulder vessels [1, 3, 4]. Unlike typical systemic reaction in wakeful humans, the reaction in narcotized rats was not too manifesting (figures 8 and 9). Nevertheless, the heart rate characteristics, as well as the R-R intervalometric diagrams, reflected the forced mechanical occlusion quite definitely. As a typical example, the comparative experimental data synchronously obtained using IRT and R-R intervalometry before, during and after forced mechanical occlusion of arteries and veins of the right hind leg of albino rat are represented in figure 9. Both types of characteristics demonstrate the threshold effect spontaneously occurring in the animal current physiological state.

As a conclusion, it should be summarized that the threshold phenomena and some other physiological processes in rats are first revealed in this work. The quantitative correlation of the data represented by two different techniques - IRT and heart rate measurement - is disclosed. On the base of various external impacts to the organism and different regimes realized in this work, in particular, using the Peltier cooler as a thermal interventional technique, it is shown that the described new combined experimental approach may be highly informative to animal and human physiology.

This research was partially supported by the Siberian Branch of the Russian Academy of Sciences (Integrative Project No. 40-B-2012).

\section{REFERENCES}

[1] Vainer B. G., "Interventional infrared thermal diagnostics in medicine and physiology", E-Book Proceedings of 11th International Conference on Quantitative InfraRed Thermography, QIRT 2012, 11-14 June, 2012, NaplesItaly. Italy: University of Naples, paper ID-340, 10 pp., 2012.

[2] Malik M., "Heart rate variability". Circulation, vol. 93, pp. 1043-1065, 1996.

[3] Vainer B. G. "Investigation of circulation in humans with the use of infrared thermography", Chapter 6 in "Circulatory System and Arterial Hypertension: Experimental Investigation, Mathematical and Computer Simulation". L. N. Ivanova , A. L. Markel , A. M. Blokhin and E. V. Mishchenko, Editors. New York: Nova Science Publishers, Inc. pp. 207-234, 2012.

[4] Vainer B. G., Markel A. L. "Imaging and quantitative characterization of bilateral vasomotor reactions in humans using high-performance thermography", QIRT10: Proceedings of the 10th edition of the Quantitative Infrared Thermography/ International Conference. July 27-30, 2010, Quebec City (Canada). X.P.V. Maldague, Editor. Canada: Universite Laval, pp. 91-94, 2010. 


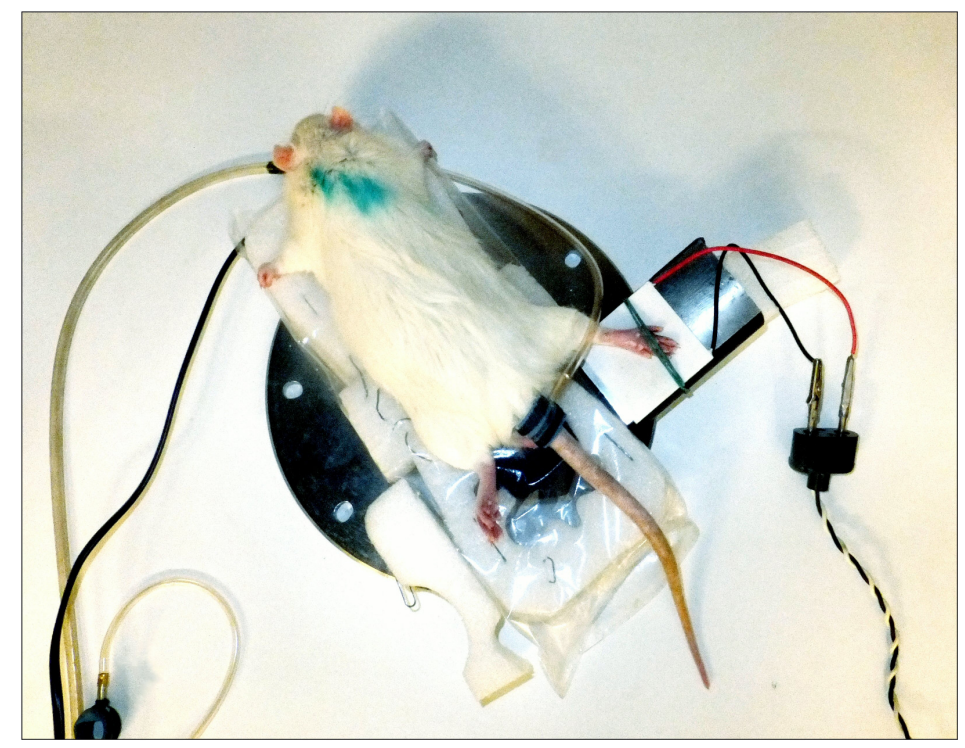

Fig. 2. Narcotized albino rat prepared for two types of external impacts during interventional IRT: mechanical occlusion of tail vessels and local thermal stress (heating or cooling). Thermal impact is performed in the right hind leg using a thermoelectric (Peltier) cooler.

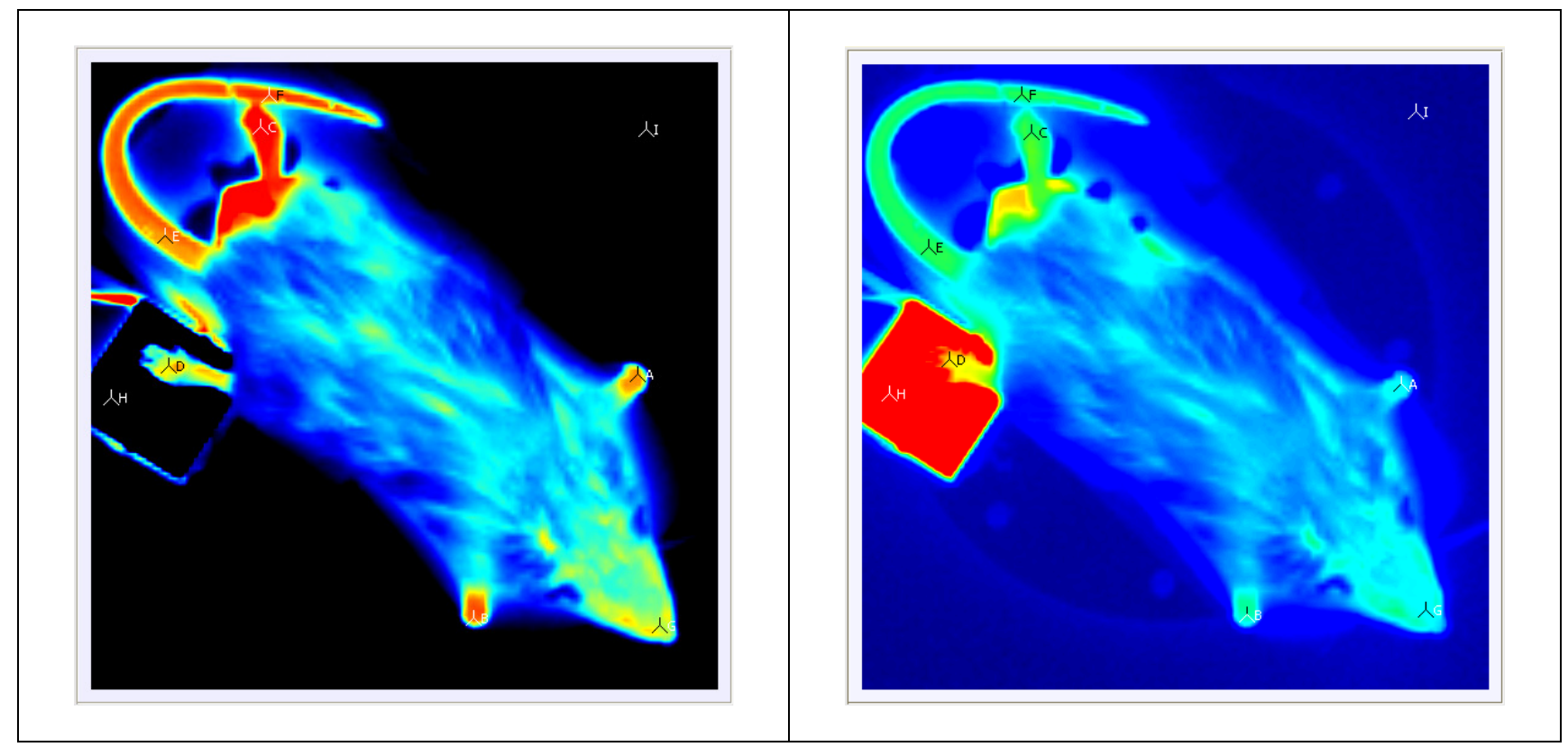

Fig. 3. Thermograms of the albino rat exposed to transient cooling (left) and heating (right) of the right hind leg with the use of a thermoelectric (Peltier) cooler. Typical checkpoints for temperature measurement are shown in the thermograms. 


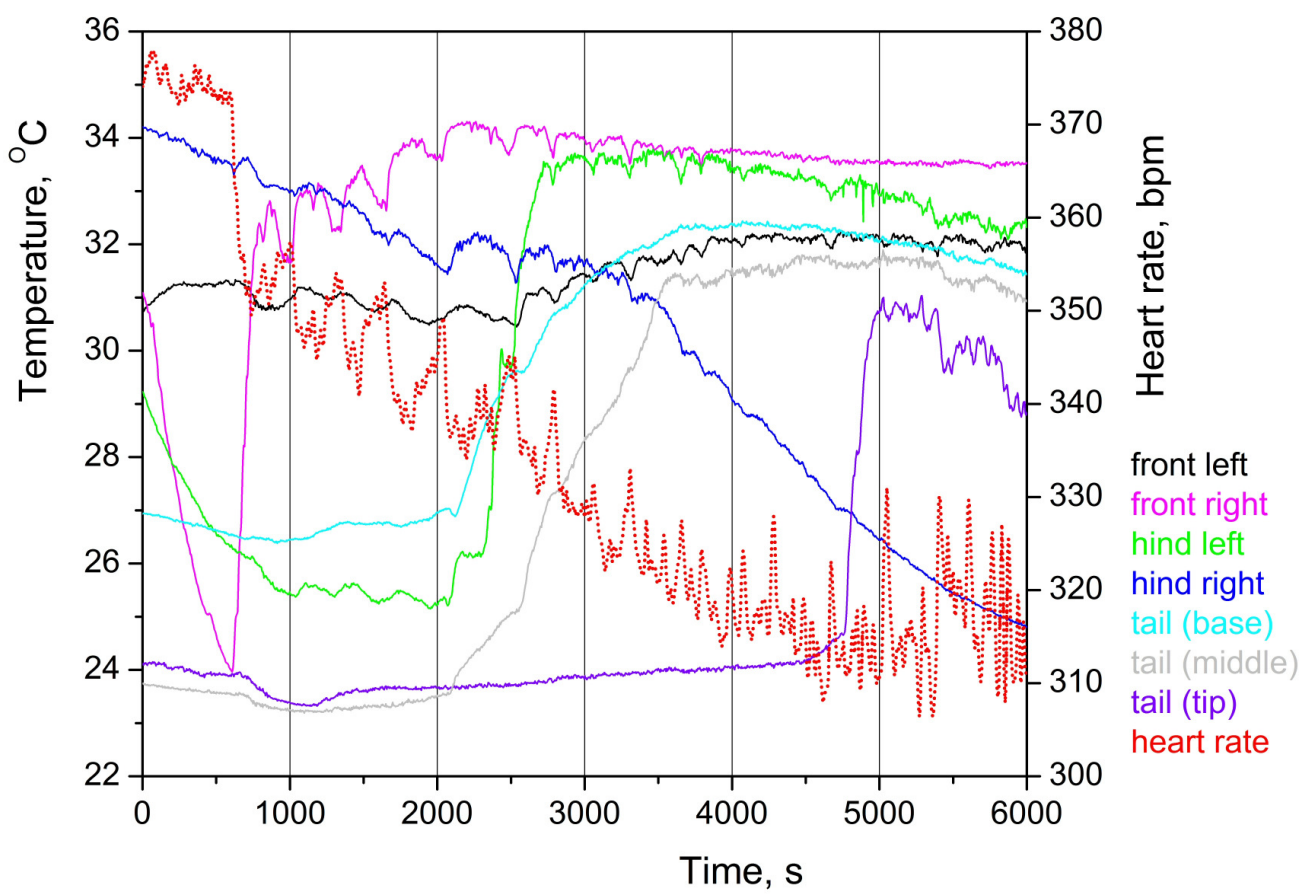

Fig. 4. Comparison of the experimental data obtained using two different methods - IRT and heart rate measurement - before any exposure of the narcotized rat to interventional impact. Rat No. 3 (green nape), 27.12.2013 (1). Checkpoints for the temperature measurement are chosen on the nonhairy parts of limbs (front left, front right, hind left, hind right) and on different parts of the tail (base, middle and tip).

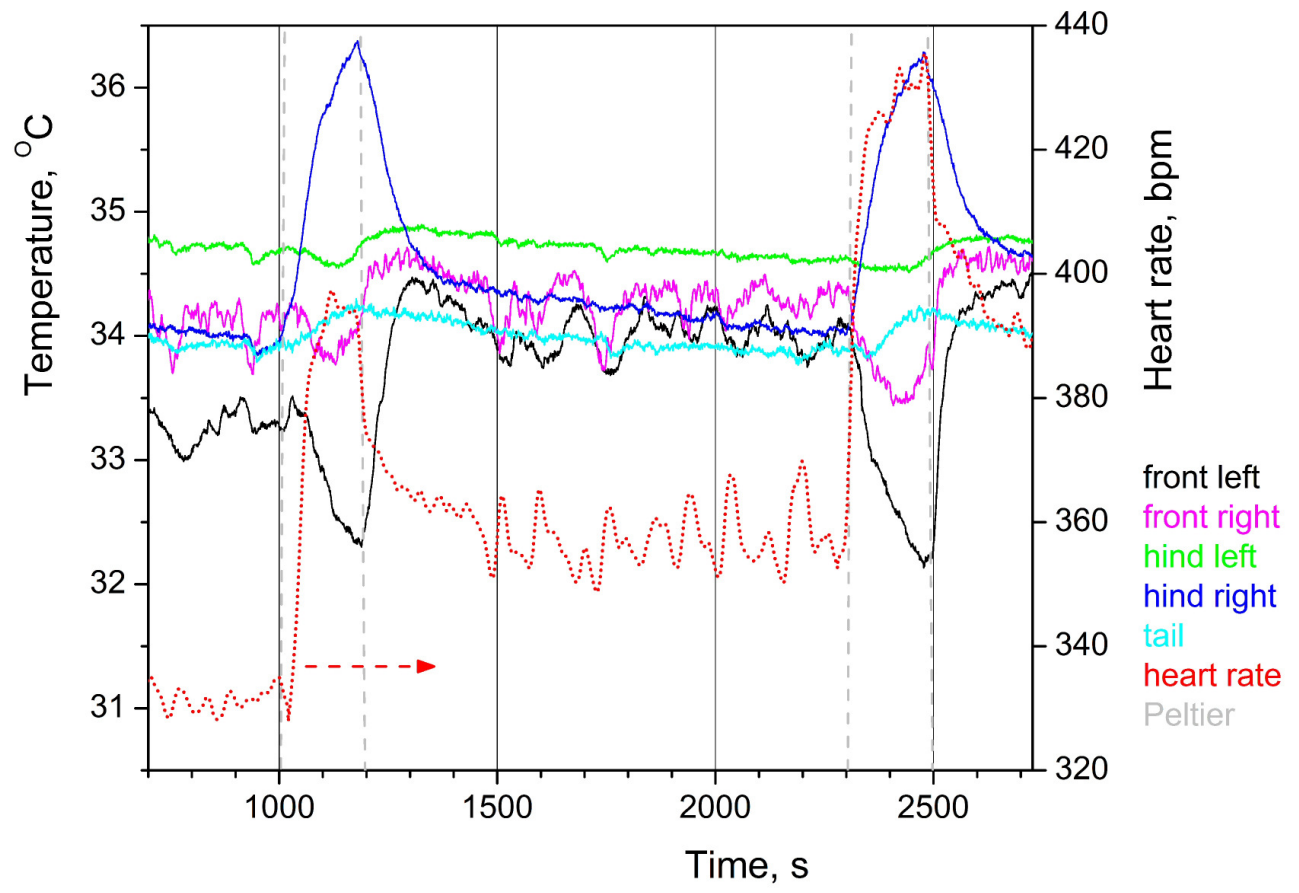

Fig. 5. Comparison of the experimental data obtained using IRT and heart rate measurement during double heating of the rat's hind right limb from the back side of Peltier cooler. Rat No. 2 (no color), 03.12 .2013 (2). The heatings were started with the time interval of $1000 \mathrm{~s}$ at $t=1000 \mathrm{~s}$ and $t=2300 \mathrm{~s}$ and prolonged for 3 minutes with maximal temperature $45^{\circ} \mathrm{C}$. Checkpoints for the temperature measurement are chosen on the nonhairy parts of limbs (front left, front right, hind left, hind right) and on the skin in the middle of the tail. 


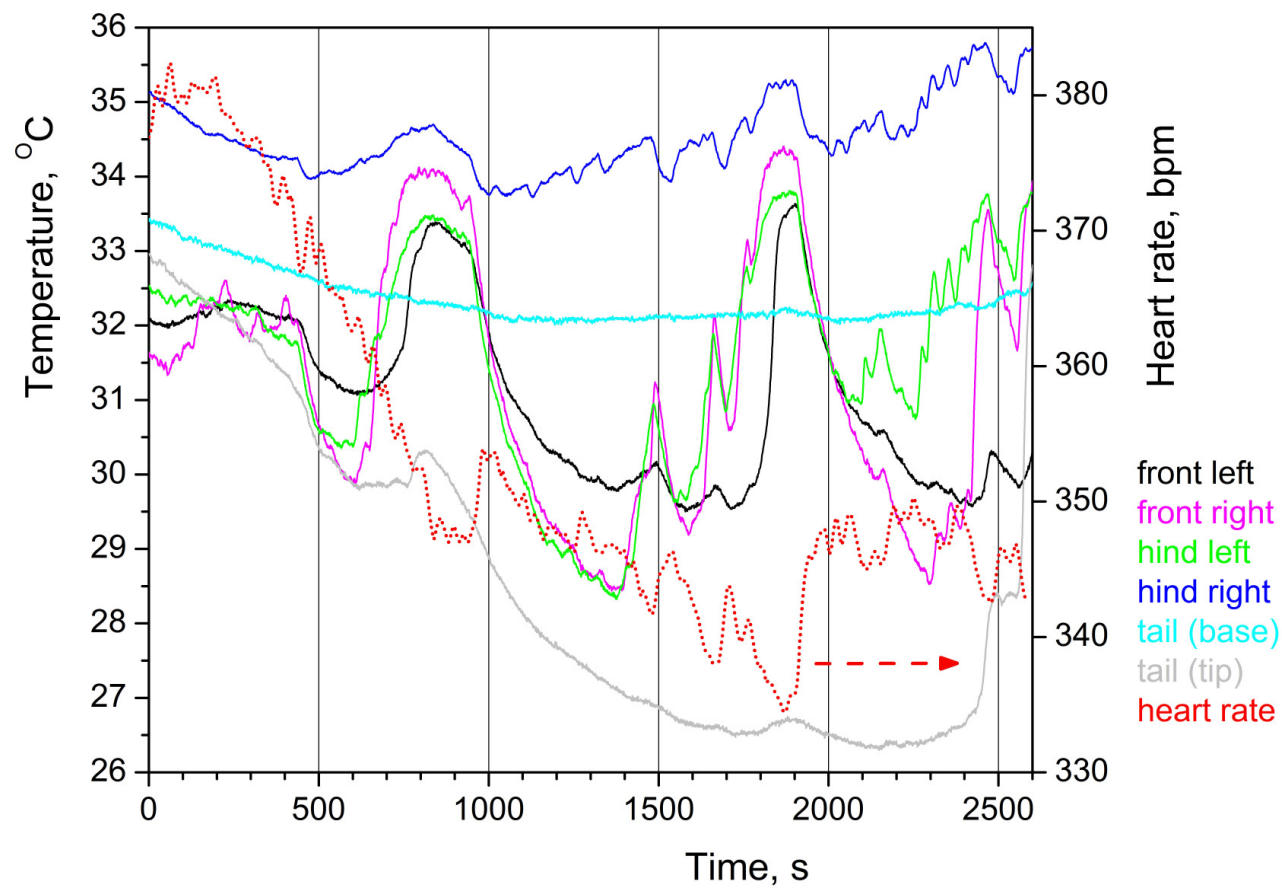

Fig. 6. Comparison of the experimental data obtained using IRT and heart rate measurement during the rat's relaxation after the double heating represented in figure 5. Rat No. 2 (no color), 03.12.2013 (3). Checkpoints for the temperature measurement are chosen on the nonhairy parts of limbs (front left, front right, hind left, hind right) and on the skin of the tail (base and tip).

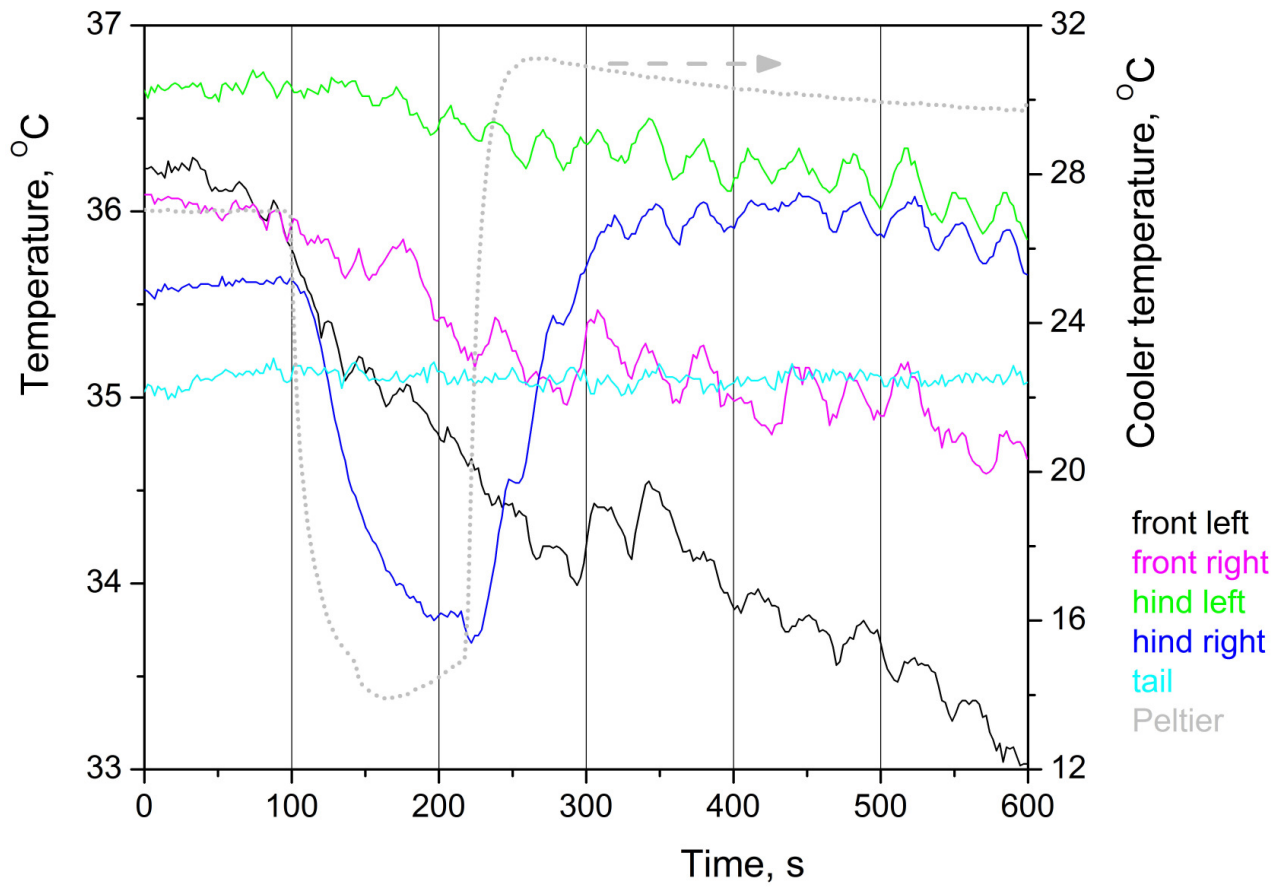

Fig. 7. Laboratory albino rat under external intervention provided in the form of a local cooling of its hind right paw from the Peltier cooler. Rat No. 3 (green nape), 06.11.2013. Checkpoints for the temperature measurement are chosen on the nonhairy parts of limbs (front left, front right, hind left, hind right) and on the skin in the middle of the tail. 


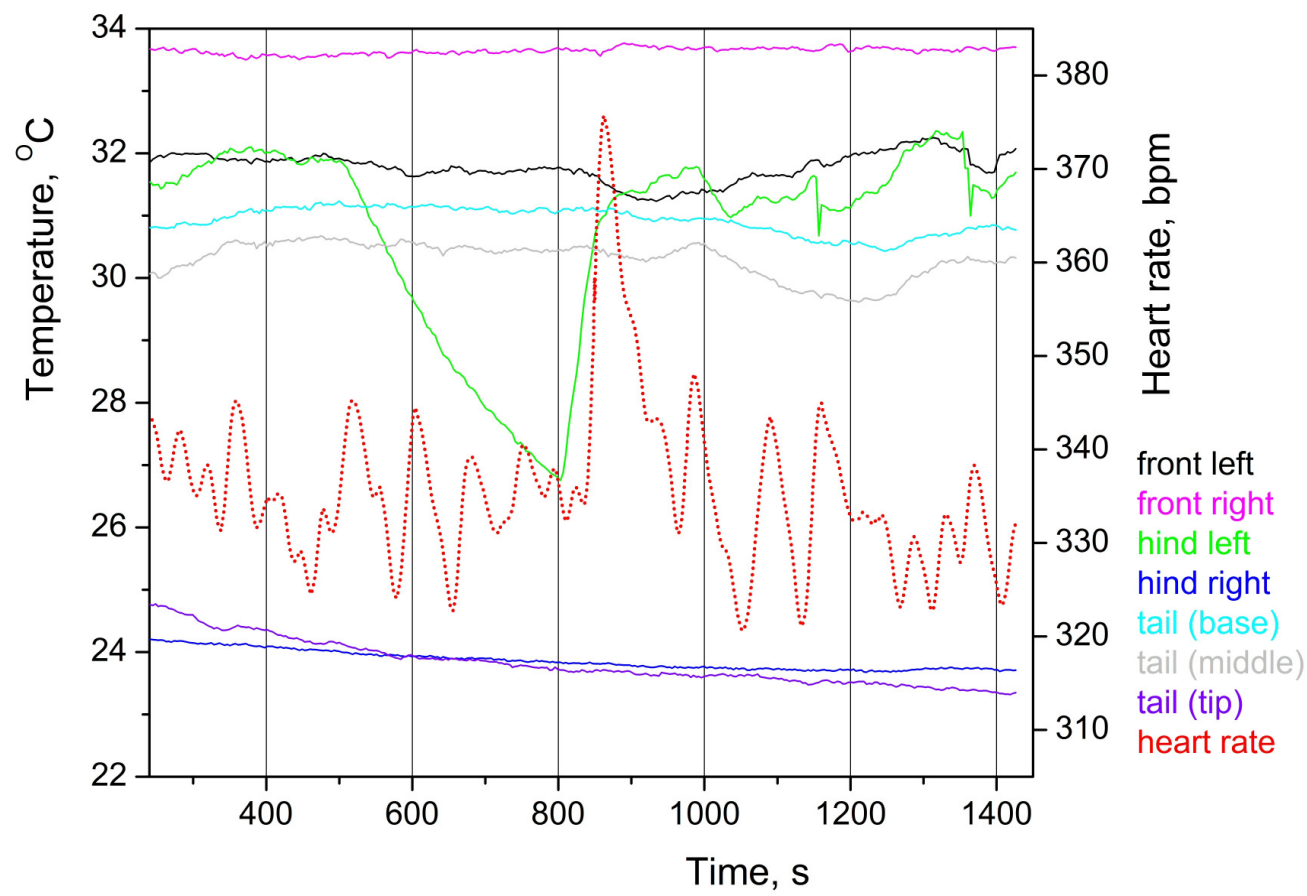

Fig. 8. Comparison of the experimental data obtained using IRT (solid lines) and heart rate measurement (dotted line) before, during and after crossclamping of arteries and veins of the animal's left hind leg. Rat No. 3 (green nape), 27.12.2013 (2).

Forced mechanical occlusion of the vessels was started at the time $t=500 \mathrm{~s}$ and prolonged for 5 minutes. Checkpoints for the temperature measurement are chosen on the nonhairy parts of limbs (front left, front right, hind left, hind right) and on different parts of the tail (base, middle and tip).

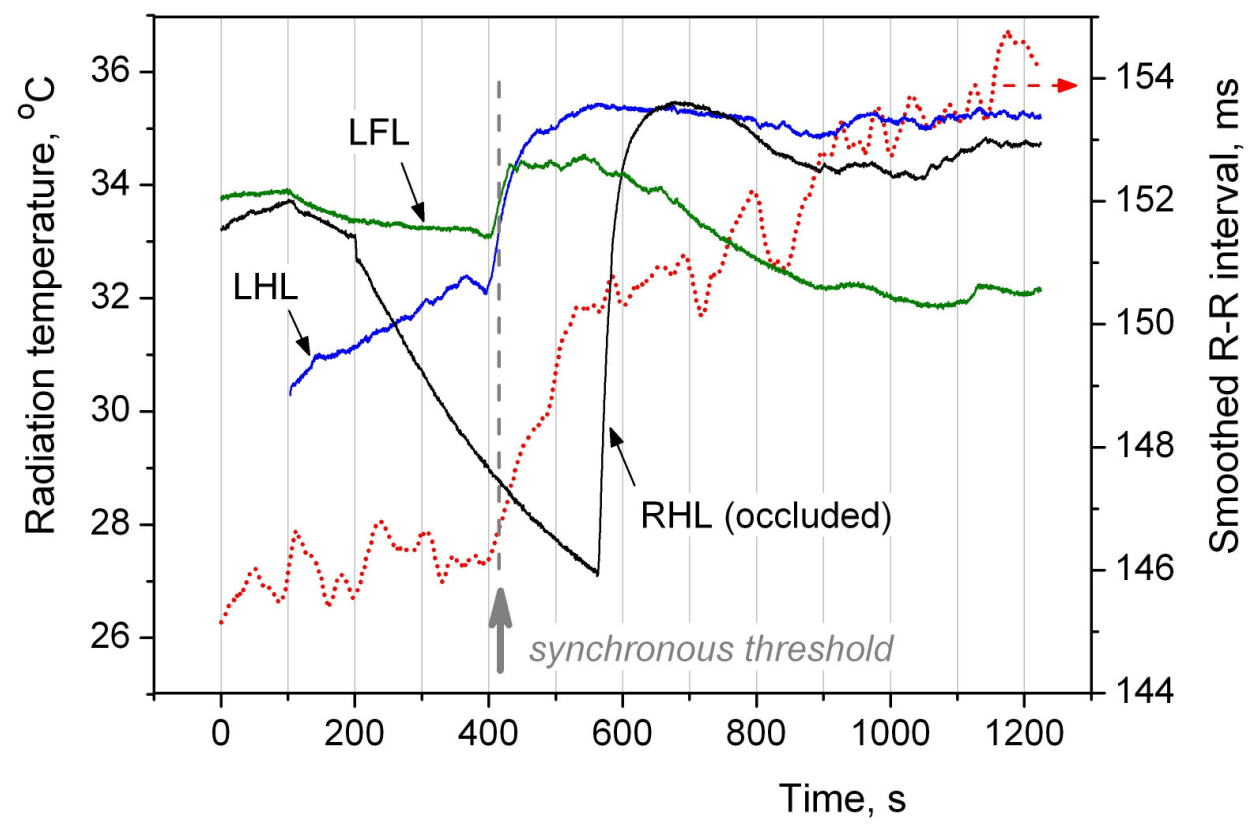

Fig. 9. Comparison of the experimental data obtained in albino rat using two different methods: IRT and $R-R$ intervalometry - before, during and after crossclamping of arteries and veins of the animal's right hind leg. Rat No. 1 (colored), 15.11.2013. Forced mechanical occlusion of the vessels was performed between 200-th and 560-th seconds of the characteristics recording. Thick arrow indicates the synchronous thermal and cardiovascular manifestations observed in remote parts of the organism.

Abbreviations: $L F L$ - left front limb, $L H L$ - left hind limb, $R H L$ - right hind limb. 